\title{
A low-voltage current conveyor using inverter-based error amplifier and its oscillator application
}

\author{
Yuh-Shyan Hwang ${ }^{1 \mathrm{a})}$, Yi-Tsen Ku ${ }^{1}$, Jiann-Jong Chen ${ }^{1}$, \\ and San-Fu Wang ${ }^{2}$ \\ ${ }^{1}$ Dept. of Electronic Eng, National Taipei University of Technology, \\ Taipei, Taiwan, R.O.C. \\ ${ }^{2}$ Dept. of Electronic Eng, Ming Chi University of Technology, \\ Taipei, Taiwan, R.O.C. \\ a)yshwang@mail.ntut.edu.tw
}

\begin{abstract}
A low-voltage second-generation current conveyor (CCII) and its oscillator application suitable for portable systems are proposed in the paper. The proposed current conveyor is based on an inverter-based low-voltage error amplifier and a current mirror. There are no on-chip capacitors in the proposed current conveyor, and it can be designed with standard CMOS digital processes and hence will reduce the cost of chip fabrication. Moreover, the architecture of the proposed circuit without cascoded MOSFET transistors is easily designed and suitable for low-voltage operation. The CCII could be operated in a very low supply voltage such as $\pm 0.5 \mathrm{~V}$. The proposed CCII has been fabricated with TSMC $0.18 \mu \mathrm{m}$ CMOS process and applied to low-voltage CCII oscillator suitable for portable systems.
\end{abstract}

Keywords: inverter-based low-voltage error amplifier, secondgeneration current conveyor

Classification: Integrated circuits

\section{References}

[1] H. P. Chen and P. L. Chu: IEICE Electron. Express 6 [2] (2009) 122.

[2] N. Herencsar and J. Koton: IEICE Electron. Express 6 [24] (2009) 1708.

[3] S. A. Mahmoud, M. A. Hashiesh and A. M. Soliman: IEEE Trans. Circuits Syst. I, Reg. Papers 52 [10] (2005) 2055.

[4] I. A. Khan, M. T. Simsim and P. Beg: Int. Conf. on Multimedia, Signal Processing and Communication Technologies (2011) 5.

[5] A. H. M. Abolila, H. F. A. Hamed and E. A. M. Hasaneen: Int. Conf. Microelectronics ICM (2010) 220.

[6] H. Moradzadeh and S. J. Azhari: IET Circuits Devices Syst. 5 (2011) 66.

[7] B. Nauta: IEEE J. Solid-State Circuits 27 (1992) 142.

[8] S. Celma, P. Martinez and A. Carlosena: Electron. Lett. 28 (1992) 443. 


\section{Introduction}

Recently, analog engineers have used current conveyors extensively to design circuits, especially, in oscillator and filter applications [1]. In analog signal processing, oscillators are important for realizing a variety of functions, and CCII is a critical component to a powerful analog CMOS oscillator [2].

In a portable system, the main power source is battery, so the mobile control analog integrated circuit needs to match small volume, low-voltage, low-power, and high output voltage and current characteristics to improve the battery life. In analog circuit design, current-mode circuits have many well-known advantages. For example, they provide high performance in speed, bandwidth and precision, and yet consume less power; thus, they have found wide applications in high-performance active circuits. Currentmode circuits do not need high voltage gain nor high precision passive components. They could be applied in all transistor designs, which make them compatible with typical digital processes [3, 4].

Most of two supply-voltage current conveyors require $\pm 0.75 \mathrm{~V}$ supply voltages or higher, but their output currents are less than $\pm 1 \mathrm{~mA}[5]$ and their oscillator amplitude are less than $50 \mathrm{mV}$. Recently, some papers proposed low supply-voltage $( \pm 0.5 \mathrm{~V})$ CCIIs, and their theories were simulated in HSPICE but was not fabricated and validated into an integrated circuit [6]. In [5], the current conveyor is designed with rail-torail differential amplifiers and its second stage is a class $A B$ cascoded current mirrors. Since the cascoded current mirrors are not low-voltage structures, the current conveyor is difficult to be designed as a low-voltage device. It is not suitable for low-voltage applications. In [6], the rail-to-rail second generation current conveyor based on quasi-floating gate transistors is presented. This circuit is using linear capacitors to couple the input signal to the gate terminal of the MOSFET transistor and its gate voltage is directly biased by a dc voltage through a diode-connection MOSFET transistor. However, this current conveyor can't be operated at low frequency, especially for dc applications. Since the current conveyor is based on the capacitor-coupled MOSFET transistors, the linear capacitors are needed. Therefore, it is not suitable for low-frequency filter and oscillator applications, and can't be fabricated with standard CMOS digital processes. Moreover, the linear capacitors will cause larger chip area. They will increase the cost of chip fabrication.

This paper proposes a current-mode active component based on an inverter-based low-voltage error amplifier [7] and a current mirror - the second-generation current conveyor (CCII). This proposed new component uses an inverter-based low-voltage error amplifier instead of class AB amplifier or other amplifier in the conventional CCII. To compare with conventional CCIIs, the proposed inverter-based CCII has low supply voltages, wide input voltages, and wide output voltages and currents. They can be operated at ultra low supply voltage $( \pm 0.5 \mathrm{~V})$. Their input voltage range can be from $\pm 5 \mathrm{uV}_{\mathrm{p}-\mathrm{p}}$ to $\pm 250 \mathrm{mV}_{\mathrm{p}-\mathrm{p}}$, which will output the same voltage and generate $\pm 0.5 \mathrm{uA}_{\mathrm{p}-\mathrm{p}}$ to $\pm 25 \mathrm{~mA}_{\mathrm{p} \text {-p }}$ current with $10 \Omega$ output load. Thus, they are highly compatible with various types of analog circuits.

This research uses CMOS characteristics and inverter-based low-voltage 
error amplifiers as well as current mirrors to construct new CCII. We design a chip of a second-generation current conveyor (CCII) for fabrication in TSMC $0.18 \mu \mathrm{m}$ CMOS processes.

\section{Circuit description}

The CCII is a three-terminal component and the ideal CCII can be described by the matrix characteristic equation as shown in (1).

$$
\left[\begin{array}{c}
i_{y} \\
v_{x} \\
i_{z}
\end{array}\right]=\left[\begin{array}{lll}
0 & 0 & 0 \\
1 & 0 & 0 \\
0 & 1 & 0
\end{array}\right]\left[\begin{array}{l}
v_{y} \\
i_{x} \\
v_{z}
\end{array}\right]
$$

Fig. 1 (a) shows the proposed low-voltage CCII circuit. The inverterbased low-voltage error amplifier [7] shown in the leftmost part of Fig. 1 (a), is a two-input and two-output low-voltage amplifier. The voltage gain of inverter-based low-voltage error amplifier $\left(A_{V d}\right)$ can be given as (2).

$$
A_{V d}=\frac{V_{o 1}-V_{o 2}}{V_{x}-V_{y}}=\frac{g_{m n 1,2}-g_{m p 1,2}}{g_{m p 1,2}+\frac{1}{r_{o p 1,2}}+\frac{1}{r_{o n 1,2}}}
$$

where $V_{o 1}$ and $V_{o 2}$ are the output voltages of $I_{n v 1}$ and $I_{n v 2}$, respectively. $V_{x}$ and $V_{y}$ are the input voltages of $I_{n v 1}$ and $I_{n v 2}$, respectively. $g_{m p 1}, g_{m p 2}, g_{m n 1}$ and $g_{m n 2}$ are the transistor's $g_{m}$ of $M_{p 1}, M_{p 2}, M_{n 1}$ and $M_{n 2}$, respectively. $r_{o p 1}, r_{o p 2}, r_{o n 1}$ and $r_{o n 2}$ are the transistor's $r_{o}$ of $M_{p 1}, M_{p 2}, M_{n 1}$ and $M_{n 2}$, respectively. Also, $g_{m p 1}=g_{m p 2}=g_{m p 1,2}, g_{m n 1}=g_{m n 2}=g_{m n 1,2}, \quad r_{o p 1}=r_{o p 2}=r_{o p 1,2}$, and $r_{o n 1}=r_{o n 2}=r_{o n 1,2}$.

The outputs of the inverter-based low-voltage error amplifier are converted into single output by a differential to single amplifier circuit, shown in the middle part of Fig. 1 (a). These two parts use closed-loop negative feedback to achieve an expected output voltage. The voltage gain of differential to single amplifier $\left(A_{V s}\right)$ can be given as (3).

$$
A_{V s}=\frac{V_{o 8}}{V_{o 6}-V_{o 3}}=g_{m p 7}\left(r_{o p 8} / / r_{o n 8}\right)
$$

where $V_{o 6}$ are the common output voltage of $I_{n v 5}$ and $I_{n v 6} . V_{o 3}$ are the common output voltage of $I_{n v 3}$ and $I_{n v 4}$. $V_{o 6}$ equals to $V_{o 1}$ and $V_{o 3}$ equals to $V_{o 2} . V_{o 8}$ is the output voltage of differential to single amplifier. $g_{m p 7}$ is the transistor's $g_{m}$ of $M_{p 7} \cdot r_{o p 8}$ and $r_{o n 8}$ are the transistor's $r_{o}$ of $M_{p 8}$ and $M_{n 8}$, respectively.

The current mirror, shown in the rightmost part of Fig. 1 (a), duplicates the output current from terminal $X$ into terminal $Z$. The voltage gain of current mirror $\left(A_{V x}\right)$ can be given as (4).

$$
A_{V x}=\frac{V_{x}}{V_{o 8}}=g_{m p 9} R_{o 9}
$$

where $V_{x}$ is the output voltage of $X$ terminal. $V_{o 8}$ is the output of differential to single amplifier. $g_{m p 9}$ is the transistor's $g_{m}$ of $M_{p 9} . R_{o 9}$ is the output resistor of $X$ terminal.

We connect the above three parts to compose the low-voltage second generation current conveyor. The total voltage gain of this composed CCII can be obtained as (5). 


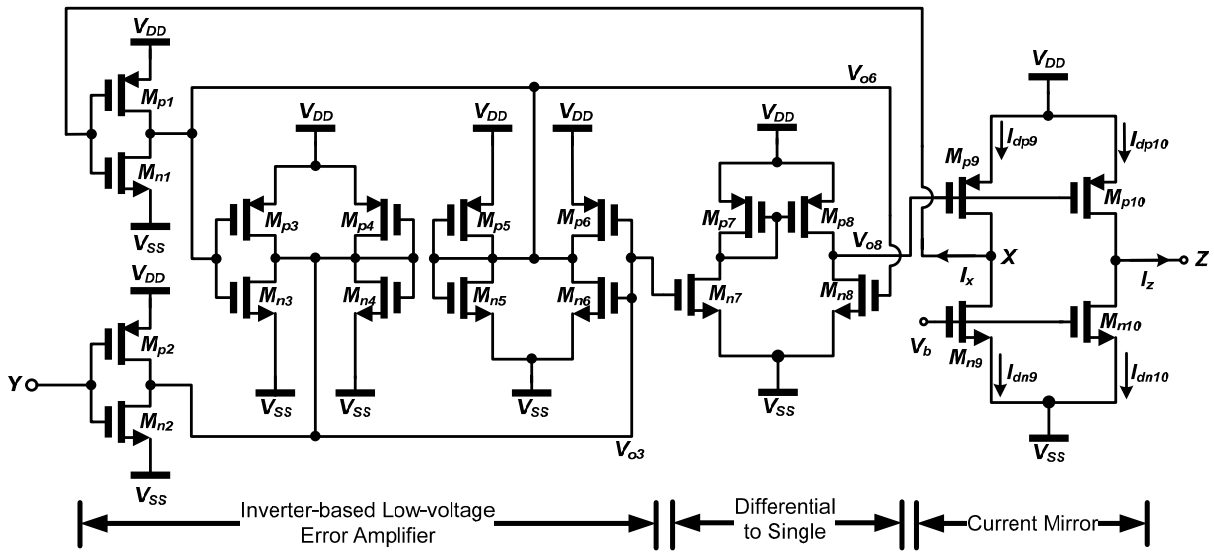

(a)

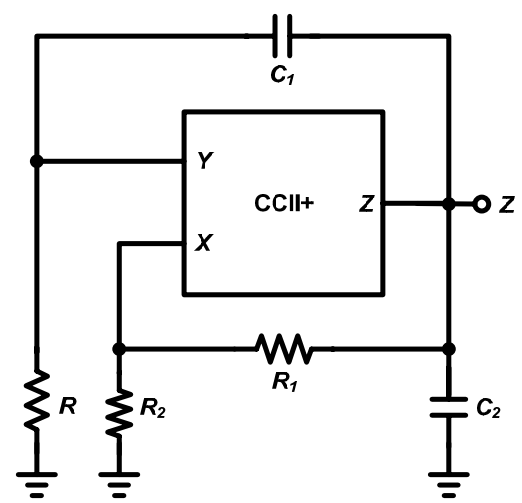

(b)

Fig. 1. (a) The inverter-based low-voltage CCII circuit (b) Single CCII oscillator

$$
A_{V t o t a l}=\frac{V_{x}}{V_{y}}=\frac{A_{V d} \times A_{V s} \times A_{V x}}{1+\left(A_{V d} \times A_{V s} \times A_{V x}\right)}
$$

The voltage gain of each part depends on transistor's $g_{m}$ and $r_{o}$. Hence, adjusting $W / L$ of transistors, we will make $A_{V d} \times A_{V s} \times A_{V x}>>1, A_{V t o t a l}$ $\approx 1$ and $V_{x} \approx V_{y}$.

For CCII, the output current of terminal $X$ and terminal $Z$ can be given as (6) and (7) respectively.

$$
\begin{gathered}
I_{x}=I_{d p 9}-I_{d n 9} \\
I_{z}=I_{d p 10}-I_{d n 10}
\end{gathered}
$$

where $I_{d p 9}, I_{d n 9}, I_{d p 10}$ and $I_{d n 10}$ are the current of transistors of $M_{p 9}, M_{n 9}$, $M_{p 10}$ and $M_{n 10}$, respectively. If the dimensions of $M_{p 9}$ and $M_{p 10}$ are equal, and the dimensions of $M_{n 9}$ and $M_{n 10}$ are equal, then $I_{z}$ equals to $I_{x}$.

To prove the functionality of this proposed CCII, we implement a CCII oscillator. Fig. 1 (b) shows the single CCII based oscillator [8]. The characteristic equation of Fig. 1 (b) is shown as (8). Its oscillation condition and frequency are given as (9) and (10), respectively.

$$
\begin{gathered}
s^{2} C_{1} C_{2}+s\left[\frac{1}{R}\left(C_{1}+C_{2}\right)-\frac{C_{1}}{R_{2}}\right]+\frac{2}{R R_{1}}=0 \\
\frac{R_{2}}{R}\left[\frac{C_{2}}{C_{1}}+1\right]=1
\end{gathered}
$$




$$
\omega_{0}=\sqrt{\frac{2}{C_{1} C_{2} R R_{1}}}
$$

\section{Experimental results}

Fig. 2 (a) shows the chip photograph of proposed CCII. The chip area of CCII is $1192 \mu \mathrm{m} \times 727 \mu \mathrm{m}$. This chip is fabricated in TSMC $0.18 \mu \mathrm{m}$ CMOS process. Dimensions of the transistors are set as $2 / 1 \mu \mathrm{m}$ for $M_{p 1}, M_{p 2}$, $1 / 1 \mu \mathrm{m}$ for $M_{n 1}, M_{n 2}, 4 / 1 \mu \mathrm{m}$ for $M_{p 3}, M_{p 4}, M_{p 5}, M_{p 6}, 1 / 1 \mu \mathrm{m}$ for $M_{n 3}, M_{n 4}$,

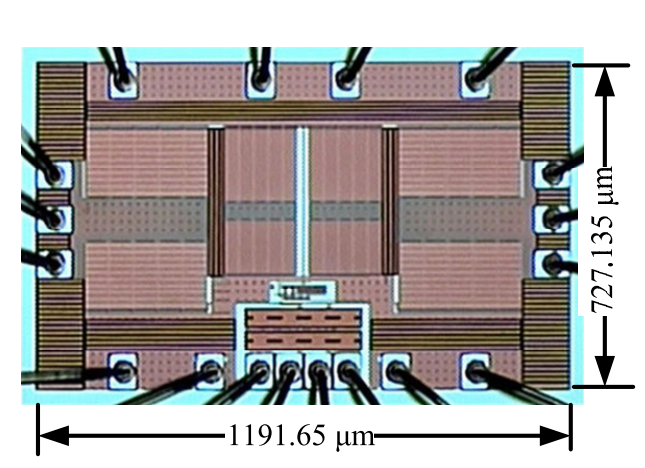

(a)

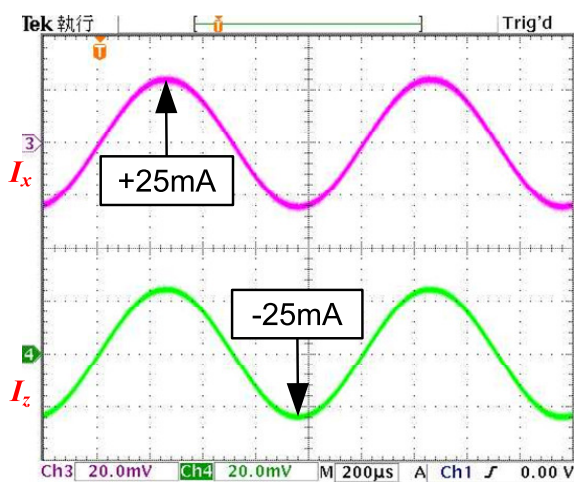

(c)

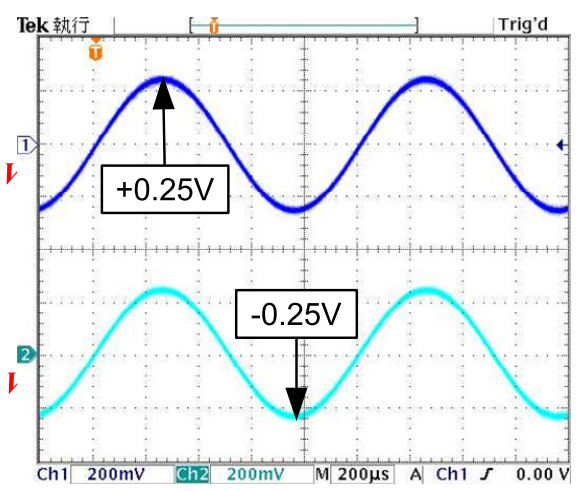

(b)

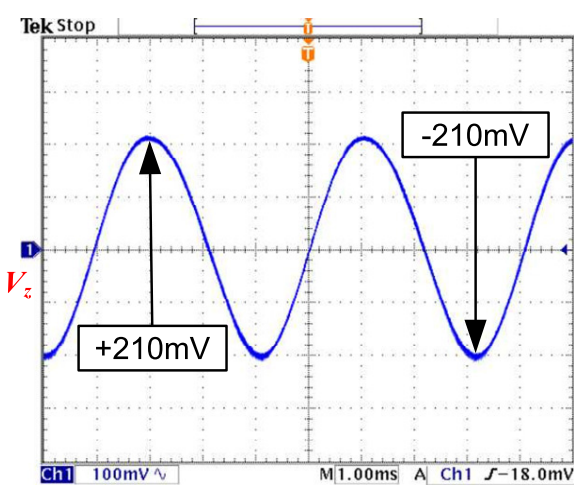

(d)

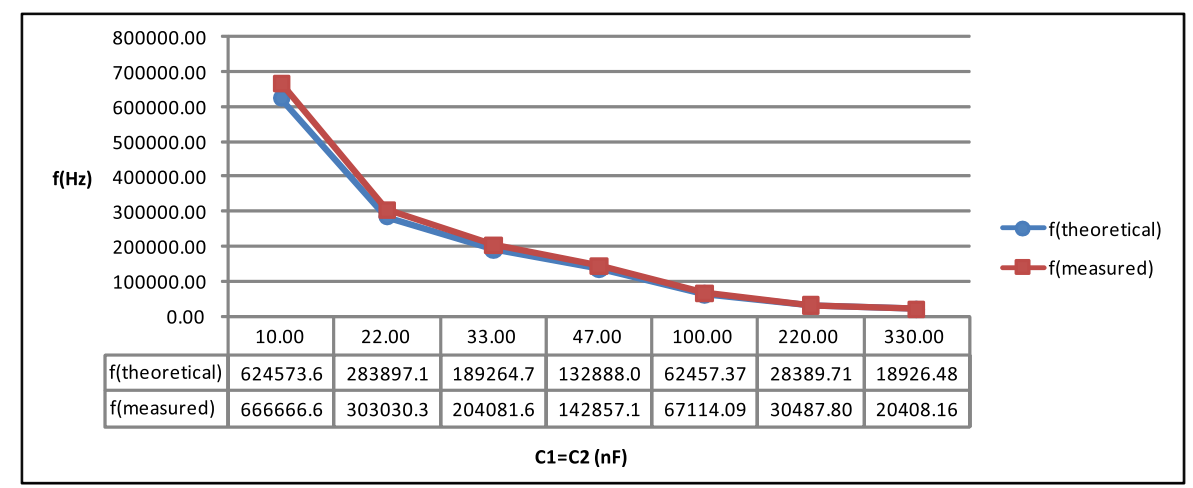

(e)

Fig. 2. (a) Chip photographs of the proposed CCII (b) The $V_{y} \& V_{x}$ waveforms of the proposed CCII (c) The $I_{x} \& I_{z}$ waveforms of the proposed CCII (d) The $V_{z}$ waveforms of the proposed CCII oscillator (e) The chart of proposed CCII oscillator with varied $C_{1}$ and $C_{2}$ 
$M_{n 5}, M_{n 6}, 3 / 1 \mu \mathrm{m}$ for $M_{p 7}, M_{p 8}, 1 / 1 \mu \mathrm{m}$ for $M_{n 7}, M_{n 8}$, and $10 / 1 \mu \mathrm{m}$ for $M_{p 9}, M_{p 10}, M_{n 9}, M_{n 10}$.

The proposed chips are operated at $\pm 0.5 \mathrm{~V}$ supply voltages. Fig. 2 (b) shows the input voltage waveform of terminal $Y$ and output voltage waveform of terminal $X$. Fig. 2 (c) shows the output current waveform of terminal $X$ and duplicate current waveform of terminal $Z$.

From the experimental waveforms, the functionalities of proposed CCII are good. The input voltage of terminal $Y$ of the proposed CCII can be raised to $\pm 0.25 \mathrm{~V}_{\mathrm{p} \text {-p }}$ with $\pm 0.5 \mathrm{~V}$ supply voltages and it generates the same output voltage at terminal $X$, which are $50 \%$ of supply voltage. Both terminals $X$ and $Z$ of CCII carry out $\pm 25 \mathrm{~mA}_{\mathrm{p} \text {-p }}$ currents with $10 \Omega$ load. Those output current are almost 10 times output current of other CCIIs. For example, the CCII output current is less than $\pm 1 \mathrm{~mA}$ in [5] and only $\pm 300 \mu \mathrm{A}$ in $[6]$

To validate the proposed CCII, we implement a CCII oscillator using Fig. 1 (b) circuit. Fig. 2 (d) shows the output waveforms of proposed CCII oscillator while operating at $\pm 0.5 \mathrm{~V}$ supply voltage. With $C_{1}=4.7 \mu \mathrm{F}$, $C_{2}=47 \mu \mathrm{F}, R_{2}=2.2 \Omega, R=24 \Omega, R_{1}=400 \Omega$, the oscillator waveform is approximated as ideal sine wave and the oscillator amplitude is $\pm 210 \mathrm{mV}_{\mathrm{p}-\mathrm{p}}$ that prove the functionality of this proposed CCII is perfectly working at low supply voltage.

Fig. 2 (e) is the chart of proposed CCII oscillator while operating at $\pm 0.5 \mathrm{~V}$ supply voltage with varied $C_{1}$ and $C_{2}\left(C_{1}=C_{2}\right)$, and fixed $R_{2}=7 \Omega$, $R=13 \Omega$ and $R_{1}=100 \Omega$. Fig. 2 (e) also shows the comparison of angular frequency of the theoretical value and experimental value. This chart proves the functionality of this proposed CCII oscillator is approximated to an ideal oscillator under $625 \mathrm{KHz}$ frequencies. The difference between the theoretical and measured results is because the theoretical value does not include the input and output impedances of the CCII. If the input and output impedances effects of CCII are considered into equations (8) to (10), the theoretical values and measured results will be closer.

Finally, we list the measurement results of the proposed current conveyor in Table I.

Table I. The measurement results of the proposed CCII

\begin{tabular}{|l|l|}
\hline Parameter & Proposed CCII \\
\hline CMOS technology & $0.18 \mu \mathrm{m}$ \\
\hline Experimental results & Measured results \\
\hline Fabricated & Yes \\
\hline Suitable for standard CMOS digital processes & Yes \\
\hline Suitable supply voltage & $\pm 0.5 \mathrm{~V}$ \\
\hline Number of transistors & 20 \\
\hline Number of linear capacitors & 0 \\
\hline Low'voltage architecture & Yes \\
\hline Current driving capability & $\pm 25 \mathrm{~mA}$ \\
\hline Voltage tracking error & $-0.0015 \mathrm{~dB}$ \\
\hline Voltage bandwidth & $75 \mathrm{MHz}$ \\
\hline Voltage performance & $99.85 \%$ \\
\hline Current tracking error & $-0.00015 \mathrm{~dB}$ \\
\hline Current bandwidth & $66 \mathrm{MHz}$ \\
\hline Current performance & $99.99 \%$ \\
\hline Power Dissipation & $25 \mathrm{~mW}$ \\
\hline
\end{tabular}




\section{Conclusion}

An inverter error amplifier-based low-voltage current conveyor is presented in this paper. In the proposed current conveyor, there are no linear capacitors and it can be designed with standard CMOS digital processes. It will reduce the cost of chip fabrication. The architecture of the proposed circuits without cascoded MOSFET transistors is easily designed and suitable for low-voltage operation. This proposed CCII can be operated in wide dynamic input range such as $\pm 250 \mathrm{mV}$ and low supply voltage such as $\pm 0.5 \mathrm{~V}$. It can work in wide input voltage $( \pm 0.25 \mathrm{~V})$, and generates wide output voltage $( \pm 0.25 \mathrm{~V})$ and large output current $( \pm 25 \mathrm{~mA})$. Its output current is almost 10 times output current of other CCIIs. For this reason, its oscillator amplitude is $\pm 210 \mathrm{mV}$ that is 4 times of other CCII oscillators. It has been fabricated with TSMC $0.18 \mu \mathrm{m}$ CMOS process. It also can be validated by low-voltage CCII oscillators. The experimental results are presented in this paper to verify the theoretical analysis and compare the proposed circuits with previous works. 